\title{
Rechtsextremismus in Deutschland
}

\section{Fakten, gesellschaftspolitische Anknüpfungspunkte, Strategien}

\author{
Artur Hertwig
}

Artur Hertwig ist Direktor beim Bundesamt für Verfassungsschutz in Köln. Internet http://www.verfassungsschutz.de
Die Zivilgesellschaft in Deutschland hat sich bisher weitgehend resistent gegen die Agitation von Rechtsextremisten gezeigt. Dennoch ist nicht zu verkennen, dass auf einzelne Bevölkerungs- und Altersgruppen abgestimmte Strategien von Rechtsextremisten punktuelle Erfolge gezeitigt haben. Rechtsextremisten haben insbesondere erkannt, dass es elementar für zukünftige Wablerfolge sein wird, eine breite gesellschaftliche Verankerung zu erreichen. An einer Umsetzung dieser Strategie arbeiten sie.

Das Thema Rechtsextremismus hat in den vergangenen Jahren für Politik und Gesellschaft eine nicht unerhebliche Bedeutung gewonnen. Die Medien beschäftigen sich ausführlich mit diesem Phänomen und gehen mit Akribie auch kleineren rechtsextremistischen Gruppierungen nach. Verfolgt man die einschlägigen Berichte über einen Zeitraum von auch nur wenigen Tagen, so ergibt sich nicht selten ein bedrohliches Bild vielfältiger rechtsextremistischer Aktivitäten: Neonazistische Vereine veranstalten Zeltlager für Kinder, finanzkräftige Aktivisten erwerben Immobilien zu Preisen in Millionenhöhe, rechtsextremistische Parlamentarier sind in Landtagen vertreten und versuchen dort, mit Reden und Anträgen zu provozieren. Die Nationaldemokratische Partei Deutschlands (NPD) stilisiert sich zur "Schutzmacht der kleinen Leute" und zieht in ostdeutschen Bundesländern in die Kommunalparlamente ein.

Geben jene Berichte tatsächlich die zutreffende Größenordnung des Phänomens wieder - und bildet die (oft vollmundige) Selbstdarstellung der Szene tatsächlich die Realität des deutschen Rechtsextremismus ab? Ferner: Ist der Rechtsextremismus - wie so oft behauptet - bereits in der Mitte der Gesellschaft angekommen? Oder stellt er (noch) ein vergleichsweise überschaubares Phänomen dar, das seine mediale Bedeutung durch die Reaktionen einer aufgeschrek- kten Öffentlichkeit gewinnt? Diese Fragen untersucht der nachfolgende Beitrag. Nach einem quantitativen Überblick steht zunächst die ideologische Ausgangsbasis der Rechtsextremisten im Mittelpunkt, wie sie von den Wortführern der NPD formuliert wird. Anschließend skizziert der Beitrag exemplarisch die Strategien von Rechtsextremisten, gesellschaftliche Akzeptanz zu erlangen und reißt die Frage an, inwieweit die Szene auch für Heranwachsende attraktiv ist. Die Darstellung mündet in die Prognose, ob und inwieweit Rechtsextremisten Aussicht darauf haben, ihre Positionen an breitere gesellschaftliche Schichten zu vermitteln.

\section{Zahlen und Fakten}

Das Spektrum des Rechtsextremismus ist spätestens seit den frühen 1990-Jahren einer der wesentlichen Beobachtungsschwerpunkte der Verfassungsschutzbehörden. Drei Segmente bestimmen im Wesentlichen sein Erscheinungsbild: die rechtsextremistischen Parteien, die Neonazis und die subkulturell geprägten gewaltbereiten Rechtsextremisten, deren Majorität immer noch von jugendlichen Skinheads gestellt wird.

Auch wenn die damalige Gewalttatenwelle abgeebbt ist, die Gefährlichkeit des Rechtsextremismus ist auch heute noch evident. In der jüngeren Vergangenheit bewegte sich die Zahl der rechtsextremistisch motivierten Gewalttaten um etwa eintausend pro Jahr. An den Zahlen alleine lässt sich ein Gefährdungspotenzial jedoch nur bedingt ablesen. Insoweit sollte uns der erhebliche Rückgang des rechtsextremistischen Personenpotenzials auf rund 31.000 (2006: 38.600) nicht in trügerischer Sicherheit wiegen. Der Rückgang liegt zum einen daran, dass die Partei »Die Republikaner « (REP) wegen des Schwindens von extremistischen Aktivitäten nicht mehr als rechtsextremistisches Personenpotenzial erfasst wird. Ein 
weiteres Sinken des Personenpotenzials resultiert aus der abnehmenden Mitgliederzahl der »Deutschen Volksunion" (DVU) auf 7.000 (2006: 8.500). Beides sind aber Organisationen, von deren Anhängerschaft zuletzt ohnehin wenig Agitation ausging. Hingegen konnte die NPD einen leichten Mitgliederzuwachs auf 7.200 (2006: 7.000) verbuchen. Das neonazistische Personenpotenzial ist nochmals leicht auf rund 4.400 (2006: 4.200) angestiegen, die Zahl gewaltbereiter Rechtsextremisten dagegen blieb in etwa konstant bei 10.000 (2006: 10.400).

\section{Die NPD als vorgebliche »Schutzmacht der kleinen Leute $«$}

Auch wenn gewaltbereite Rechtsextremisten und Neonazis mit insgesamt mehr als 14.000 Anhängern den größeren Teil des rechtsextremistischen Gesamtpotenzials in Deutschland stellen, ist die NPD mit ihren (nur) 7.200 Mitgliedern die einzige rechtsextremistische Kraft, die langfristig und planmäßig versucht, sich in der Gesellschaft zu verankern. Ihr Ziel ist es, über Wählerzustimmung in Parlamente auf kommunaler, Landes- und zuletzt Bundesebene einzuziehen, um dort politische Entscheidungen in ihrem Sinne zu beeinflussen. Führende NPD-Ideologen haben bereits vor einiger Zeit erkannt, dass Wahlerfolgen eine Verankerung der Partei in der Gesellschaft vorausgehen muss. Dabei sieht sich die NPD von den politischen, gesellschaftlichen und sozialen Entwicklungen in Deutschland begünstigt.

Jürgen Gansel, häufig zitierter Vordenker der Partei und Abgeordneter der NPD im Sächsischen Landtag, vermeinte schon 2006 einen "modernen Nationalismus « zu erkennen, der "unaufhaltsam in die Mitte der Gesellschaft « einsickere. Dieser Nationalismus habe »das Sektiererhafte und Bürgerschreckhafte früherer Zeiten weit hinter sich gelassen « und docke »erfolgreich an die Alltagsrealität der Menschen an «. In Teilen "Mitteldeutschlands " hätten "Nationalisten mit völkisch aufgeladenen Sozialthemen bereits die Meinungsführerschaft errungen, deren Rückeroberung den Systemkräften nicht mehr gelingen « werde. Die bislang noch ausgebliebenen flächendeckenden Wahlerfolge rechtsextremistischer Par- teien seien kein Indiz gegen diese These, da »nationales Alltagsbewußtsein (...) zwar oft noch kein nationales Stimmverhalten nach sich « ziehe, aber »das eine im Sinne der kulturellen Hegemoniegewinnung die unabdingbare Voraussetzung für das andere « sei. (1) Diese Auffassung zieht sich wie ein roter Faden durch die Argumentationsmuster von Rechtsextremisten und besonders der NPD.

Besonders ausgeprägt - so Gansel - sei die Empfänglichkeit der Menschen für rechtsextremistische Ideologien in den neuen Bundesländern. Dort finde bereits »eine geräuschlose völkische Graswurzelrevolution statt «, über die es Rechtsextremisten gelinge, »mit einem moderaten Ton, zivilem Auftreten und alltagsnahen Themen (...) vielerorts zum integralen Be-
Mit der Ausrichtung auf einen "völkisch aufgeladenen « Sozialismus begeben sich Rechtsextremisten zumindest thematisch auf eher »linkes « Terrain. Bei der Werbung um die Gunst von Protestwählern konkurriert die NPD dabei in direktem Maße mit den auf der linken Seite des Parteienspektrums angesiedelten Parteien. Nach Meinung - und wohl gleichzeitig Hoffnung - von NPD-Funktionären hätten »SPD, Grüne, WASG und Linkspartei (...) dem Nationalismus das Feld der sozialen Frage aber schon längst überlassen «, dies aus »Saturiertheit, Machtbesessenheit und Überzeugung «. In Wahlkämpfen habe die Linke zwar noch deren bekannte Sozialrhetorik im Repertoire, aber inhaltlich das Themenfeld bereits geräumt. Dies führe dazu,

\section{»Angestrebt wird die >Nationalisierung des Sozialen und eine >Ethnisierung der sozialen Frage«"}

standteil des gesellschaftlichen Lebens zu werden ", während sich die »Systemkräfte « dem Volk immer mehr entfremdeten und »in der Lebenswelt der von ihnen tief enttäuschten Durchschnittsbürger immer weniger « vorkämen. (2) Dabei spielt für die von Gansel angemahnte »kulturelle Hegemoniegewinnung " vor allem ein Thema eine zentrale Rolle: die so genannte "Soziale Frage«. Sie bereite das " politische Schlachtfeld, auf dem sich die Zukunft der nationalen Opposition und damit des deutschen Volkes « entscheide. Soziale Themen, die »die gesellschaftlichen Verhältnisse zum Tanzen bringen " könnten, lägen »auf der Straße«; sie müssten von der Partei nur »beherzt, seriös und volksnah aufgegriffen «werden. Dies könne die NPD dadurch erreichen, dass sie sich als "Schutzmacht der kleinen Leute « positioniere, indem sie glaubhaft für "soziale Gerechtigkeit in dieser sozial aus allen Fugen geratenen Republik der Neoliberalisten « eintrete. Dem Bürger müsse eingeschärft werden, dass »die Volksgemeinschaft in der Globalisierungsära die einzig denkbare Schutz- und Solidargemeinschaft « sei. Ohne einen Nationalstaat könne es keinen Sozialstaat geben und ohne nationale Solidarität keine soziale Solidarität. (3) dass Rechtsextremisten »zukünftig noch leichter die Position des Antikapitalismus aus den Traditionsbeständen der Linken herausbrechen und mit nationalen Inhalten aufladen « könnten. Mit der »Nationalisierung des Sozialen « gehe auch eine »Ethnisierung der sozialen Frage« einher. (4)

Im Kampf um die Meinungsführung auf dem Feld der »Sozialen Frage « agitieren Rechtsextremisten besonders aggressiv gegen die Partei »DIE LINKE.«, die sich in Teilbereichen um die gleiche Wählerklientel wie die NPD bemüht. Für beide Parteien, die den Großteil ihrer Wähler über soziale Themen erreichen, ist die Glaubwürdigkeit ihrer Argumentation auf diesem Feld essenziell. Rechtsextremisten versuchen daher, der Partei »DIE LINKE. « die Authentizität abzusprechen, indem sie ihr eine systemstabilisierende Rolle zuschreiben. Jürgen Gansel unterstellte der Konkurrenzpartei zwei Aufgaben, die dieser von der etablierten Politik zugewiesen worden seien. Zum einen sichere "DIE LINKE. " der bundesdeutschen Gesamtlinken die strukturelle Regierungsfähigkeit, zum anderen sorge sie für eine Eindämmung der NPD, indem sie den "gerechten Volkszorn der sozial Ausgegrenzten und Abstiegsbedrohten zu 
kanalisieren und seine Wendung nach rechts (...) verhindern « solle. Mögliche temporäre Erfolge der Partei »DIE LINKE. « zu Lasten der NPD seien dadurch zwar möglich. Allerdings erzeuge »DIE LINKE. « durch eine »tabubrechende Irrlichterei zwischen Links und Rechts « in der Bevölkerung »national-soziale Wallungen ", die ein "politisch aufgewühltes und protestbereites Wahlvolk « hinterließen, das für die etablierten Parteien verloren sei. In einem solchen Umfeld sei es dann auch für Rechtsextremisten möglich, Wahlerfolge zu erzielen. (5)

\section{Strategien zur Anknüpfung an die Gesellschaft}

Bei der Suche nach Anbindung an die bürgerliche Gesellschaft agieren Rechtsextremisten mit einer doppelten strategischen Ausrichtung. Zunächst spielt die parlamentarische Arbeit eine wichtige Rolle. So wachsen der NPD, die seit 2004 in Sachsen und seit 2006 in Mecklenburg-Vorpommern in den Landesparlamenten vertreten ist sowie mittlerweile auch über Kommunalmandate in Berlin, Brandenburg, Hessen, MecklenburgVorpommern, Niedersachsen, Nordrhein-Westfalen, im Saarland, in Sachsen und in Sachsen-Anhalt verfügt, finanzielle Ressourcen aus ihrer parlamentarischen Arbeit zu. Die Mandate sichern zudem die bundesweite öffentliche Aufmerksamkeit, was einen weiteren elementaren Beitrag zur Existenzsicherung der Partei bedeutet. Durch gezielte Tabubrüche - etwa durch den von Jürgen Gansel geprägten Begriff des »Bombenholocaust « (6) oder der vom Fraktionsvorsitzenden Holger Apfel geprägten diffamierenden Rede von den » Wohlstandsnegern « (7) - hat sich die NPD-Fraktion vor allem in Sachsen zumindest temporär Aufmerksamkeit verschafft.

Daneben wurde im Umfeld der sächsischen NPD-Fraktion mit der »Dresdner Schule« eine Denkfabrik der Partei gegründet, die - bisher allerdings nur mit mäßigem Erfolg - versucht, die politische Diskussion im Land über eine ideologisch unterfütterte, auf intellektuellem Niveau stehende politische Grundlagenarbeit zu indoktrinieren.

Dennoch bleibt die tatsächliche politische Wirkung der NPD eher gering; dies trotz aller echter und vermeintlicher
Eklats und Skandale, die die Wortmeldungen von Abgeordneten der Partei in den Parlamenten von Dresden und Schwerin nach sich zogen - immerhin kassierten die Abgeordneten der Schweriner NPD-Fraktion im Zeitraum zwischen Oktober 2006 und Juli 200739 Ordnungsrufe und vier Sitzungsverweise. (8) $\mathrm{Zu}$ konsequent formiert sich mittlerweile die Ablehnung der NPD durch die demokratischen Parteien. In beiden Landtagen ist es Usus geworden, Anträge und parlamentarische Initiativen der NPD abzulehnen, und zwar geschlossen mit den Stimmen aller anderen Fraktionen. Bei unvermeidlichen Entgegnungen auf NPD-Anträge spricht ein Abgeordneter stellvertretend für alle anderen Fraktionen. (9) Dies bedeutet - auch im $\mathrm{Zu}$ sammenspiel mit dem weitgehenden medialen Boykott der NPD-Parlamentsarbeit - im Umkehrschluss, dass die Öffentlichkeitswirkung von Rechtsextremisten gering bleibt. Daher suchen sie auch auf der lokalen Ebene nach Verankerung, indem sie dort eine Vielzahl von Aktivitäten an den Tag legen. Einige durchaus kreative Aktionen - seien nachfolgend exemplarisch beschrieben.

So besteht eine enge Zusammenarbeit zwischen den Fraktionen in Mecklenburg-Vorpommern und Sachsen und den lokalen Bürgerbüros von NPD-Abgeordneten in ihren Wahlkreisen. Seit der Landtagswahl in Sachsen 2004 und besonders nach der Landtagswahl in Mekklenburg-Vorpommern 2006 haben etliche NPD-Abgeordnete derartige Anlaufstellen eröffnet, in denen sie den Bürgen persönliche Beratungen - etwa beim Ausfüllen von Anträgen auf Arbeitslosengeld und Sozialhilfe - anbieten und Fragen beantworten.

Auch geben Rechtsextremisten regelmäßige regionale Informationsbroschüren oder Lokalzeitungen heraus, um die lokale Verankerung zu demonstrieren.

Brandenburger NPD-Kreisverbände verteilen bereits seit einiger Zeit - und damit unabhängig von den im September 2008 stattgefundenen Kommunalwahlen - Faltbroschüren mit Titeln wie »Havelland-Stimme « oder »Märkische Stimme «, in denen neben allgemeinen politischen Themen auch konkrete lokale Bezüge im Sinne der Partei kommentiert werden. Während sich diese Broschüren eher an potenzielle Wähler richten, haben NPDUntergliederungen bereits professionell aufgemachte Publikationen für Jugendliche herausgegeben, die einer indirekten politischen Beeinflussung dienen sollen. Größere Aufmerksamkeit erlangten in diesem Zusammenhang zwei Ausgaben der vom Landesverband Sachsen der NPD-Jugendorganisation »Junge Nationaldemokraten « (JN) edierten Schülerzeitung »perplex «. Diese wurden im September und Dezember 2007 - nach Aussage der Verantwortlichen in einer Auflage von mehreren zehntausend Exemplaren - an Schulen, Jugendclubs und Bushaltestellen an Jugendliche verteilt.

Neben Publikationen haben NPD und Neonazis in der Vergangenheit auch Tonträger mit der Absicht verteilt, Jugendliche auch ohne offensichtliche offensive politische Werbung zu gewinnen. Inspiriert durch den (letztlich gescheiterten) Versuch von Neonazis, im Rahmen einer so genannten »Schulhof-Kampagne« im Jahr 2004 insgesamt 50.000 Musik-CDs an Schulen und Jugendtreffpunkten zu verbreiten, übernahm die NPD im sächsischen Landtagswahlkampf 2004 das Konzept und produzierte ihrerseits einen Tonträger mit dem Titel »Schnauze voll? - Wahltag ist Zahltag «. Die CDs wurden im Wahlkampf im Umfeld von Schulen verteilt. Auch bei späteren Wahlen - etwa 2005 zur Landtagswahl in Schleswig-Holstein, zur Bundestagswahl 2005 sowie im Rahmen der Landtags- bzw. Senatswahlen in Mecklenburg-Vorpommern und Berlin im Jahr 2006 - tauchten entsprechende Tonträger in leicht veränderter Machart auf. Sie trugen Titel wie "Schulhof-CD - Hier kommt der Schrecken aller linken Spießer und Pauker « oder »Rebellion im Klassenzimmer - Franken rockt «.

Die Fokussierung auf Jugendliche kommt nicht von ungefähr. Aus strategischen Gründen misst die NPD der - maßgeblich von ihrer Jugendorganisation getragenen - Jugendarbeit einen hohen Stellenwert zu. Sie verknüpft dabei die Werbung unter Jugendlichen mit dem Bemühen um weitere lokale Verankerung sowie um Resonanz in den Medien. Das Konzept, kostenlose Werbemedien an Jugendliche und Jungerwachsene zu verteilen, um langfristige politische Bindungen zu schaffen, korrespondiert mit den Erfolgen, den die NPD in der Vergangenheit bei jungen Wählern erringen konnte. Die Analyse von Wahlen der letzten Jahre auf Bundes- und Landesebene zeigt, dass rechtsextremistische Parteien - außer von 
Arbeitslosen - zu einem überdurchschnittlichen Prozentsatz von Erst- und Jungwählern Stimmen erhalten. So wählten etwa bei der Landtagswahl am 17. September 2006 in Mecklenburg-Vorpommern 17 Prozent der 18- bis 24-jährigen Wähler die NPD, die im Gesamtergebnis dagegen nur 7,3 Prozent aller abgegebenen Stimmen erringen konnte. (10)

Auch Demonstrationen sollen eine andauernde Präsenz von Rechtsextremisten im öffentlichen Raum sichern. Während größere Aufmärsche mit mehreren tausend Teilnehmern eher der medialen (Provokations-) Wirkung dienen, versuchen Rechtsextremisten - und hier sowohl Neonazis als auch die NPD - im lokalen Bereich Themen zu besetzen, die von örtlicher Verankerung zeugen.

So demonstrierten rund 70 NPD-Anhänger am 21. Juni 2008 im brandenburgischen Joachimsthal unter dem Motto "Sicherheit, Recht, Ordnung - Keine Gnade für die Täter " am Wohnsitz eines nach Verbüßung seiner Freiheitsstrafe entlassenen Sexualstraftäters. Dabei konnte der verantwortliche Kreisverband Barnim-Uckermark nach eigener Darstellung durchaus Zuspruch aus der Bevölkerung für sich verbuchen. (11) Eine Vielzahl von weiteren öffentlichen Veranstaltungen wie Mahnwachen, Informationsstände oder Flugblattverteilungen flankiert derartige Parteiaktivitäten und sind oft mit sozialen Themen unterfüttert. Derzeit führt die NPD eine bundesweite Kampagne unter dem Tenor »Sozial geht nur national «. Eine solch flächendeckende Präsenz hält die NPD allerdings wegen ihrer oft mangelhaften personellen Ressourcen nur in einigen Regionen Deutschlands aufrecht.

\section{Jugendspezifische Aspekte}

Angesichts von mehr als 80 Millionen Menschen in der Bundesrepublik ist das erwähnte rechtsextremistische Personenpotenzial natürlich kein Beleg für die gesellschaftliche Relevanz von Rechtsextremisten. Doch wie am Beispiel der NPD deutlich wurde, gibt es Bereiche, die eine größere politische und sicherheitsbehördliche Relevanz entfalten als dies eine zunächst geringe quantitative Präsenz vermuten lässt. Dies gilt auch für die so genannten "Autonomen Nationalisten « (AN), die in enger Anlehnung an Klei- dung und Habitus linksextremistischer Autonomer vor allem bei Demonstrationen als "Schwarzer Block " in aggressivmilitanter Art und Weise in Erscheinung treten. Auch wenn das Bundesamt für Verfassungsschutz die Anzahl der Angehörigen dieser Strömung des Neonationalsozialismus auf nur etwa ein Zehntel des Potenzials der Neonazis, also etwa auf 400 bis 500 Personen schätzt, stellen die »Autonomen Nationalisten « durchaus ein Bindeglied zwischen ideologisch fundierten Rechtsextremisten und bürgerlichen Jugendlichen dar. Über den Aktionismus der "Autonomen Nationalisten « und deren habituelle Modernität können Jugendliche und Jungerwachsene durchaus an rechtsextremistische Einstellungen herangeführt werden. Gleiches gilt auch für den Bereich der rechtsextremistischen Musik, deren Konsum aus Jugendlichen zwar keine gefestigten Rechtsextremisten macht, aber durchaus deren Hemmschwellen gegenüber rechtsextremistischer Ideologie beseitigen kann.

\section{Fazit}

Ersichtlich sind die Anknüpfungspunkte für Rechtsextremisten an die Gesellschaft vielfältig. Auf einzelne Bevölkerungs- und Altersgruppen zielgerichtet abgestimmte Strategien bieten zumindest die Möglichkeit einer punktuellen gesellschaftlichen Anbindung, wie sie in einigen Regionen unseres Landes bereits zu sehen ist. Rechtsextremisten haben erkannt, dass es elementar für zukünftige Wahlerfolge sein wird, eine breite gesellschaftliche Verankerung zu erreichen. An einer Umsetzung dieser Strategie arbeiten sie, und dies nicht nur über die Aktivitäten der NPD.

Trotz aller Werbungsversuche von Rechtsextremisten um Sympathien in der bürgerlichen Gesellschaft - und so erfolgversprechend sie im Einzelfall auch sein mögen: Eine akute Bedrohung der freiheitlichen demokratischen Grundordnung durch Rechtsextremisten jedweder Couleur gibt es derzeit nicht. Dies machen allein die Größenordnungen der Mitglied- und Anhängerschaften rechtsextremistischer Spektren und Organisationen deutlich. Ebenso existiert keine kulturelle Hegemonie, die es Rechtsextremisten erlauben würde, inhaltlich auf aktuelle politische oder gesellschaftliche $\mathrm{Di}$ - skussionen Einfluss zu nehmen. Allem Anschein nach wird dieses Ziel auch mittelfristig nicht erreicht werden. Die Zivilgesellschaft hat sich bisher erfreulich resistent gegen die Agitation von Rechtsextremisten gezeigt; es ist zu hoffen, dass dies auch zukünftig so bleiben wird.

\section{Anmerkungen}

(1) Gansel, Jürgen W.: Der Marsch in die Mitte des Volkes, in: www.npd.de vom 27.03.2006; abgerufen am 01.08.2008.

(2) Gansel, Jürgen W.: Die nationale Achse Dresden - Berlin - Schwerin, in: www.npd.de vom 21.09.2006; abgerufen am 01.08.2008.

(3) Gansel, Jürgen W.: Die Schutzmacht der kleinen Leute, in: »Deutsche Stimme " Nr. 08/08 vom August 2008, S. 13.

(4) Gansel, Jürgen W.: Der Abschied der Linken von der sozialen Frage, in: »Deutsche Stimme « Nr. 12/06 vom Dezember 2006, S. 19.

(5) Gansel, Jürgen: »Die Linke« als letzte Auffangformation des Systems, in: »Deutsche Stimme« Nr. 08/07 vom August 2007, S. 23.

(6) So Gansel am 21. Januar 2005 in einer von der NPD beantragten Aktuellen Stunde des Dresdner Landtags zum Jahrestag der Bombardierung Dresdens.

(7) Holger Apfel in einer Rede vor dem sächsischen Landtag zur Debatte um das Zuwanderungsgesetz am 8. Mai 2007.

(8) Niemann, Laura: Die NPD im Landtag von Mecklenburg-Vorpommern. Ihre Parlamentsarbeit im ersten Jahr, in: Universität Greifswald (Hg.): Demokratie Politik. Politikwissenschaftliche Arbeitspapiere aus dem Arbeitsbereich Politische Theorie und Ideengeschichte, Heft 4, Greifswald 2008, S. 56.

(9) Ebd., S. 58.

(10) Infratest Dimap (Hg.): Wahlreport. Landtagswahl Mecklenburg-Vorpommern 17. September 2006, Berlin 2006, S. 48.

(11) Internet http://npd-barnim.de/blog/ index.php/2008/06/22/demonstrationsbericht-joachimsthal-barnimam- 21062008; abgerufen am 19.08.2008 